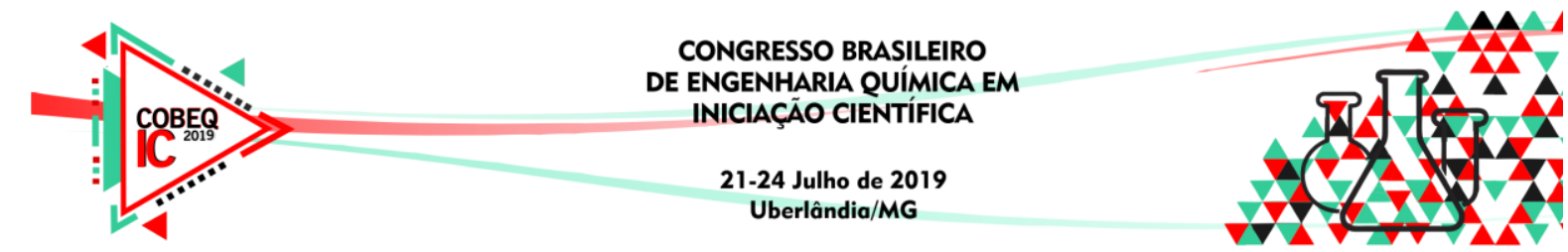

\title{
DESENVOLVIMENTO DE METODOLOGIAS ANALÍTICAS PARA A ANÁLISE DO AGENTE REDUTOR LÍQUIDO AUTOMOTIVO
}

\author{
M. H. R. BARBOSA ${ }^{1}$, J. P. DA SILVA ${ }^{2}$ e T. M. POPIOLSKI ${ }^{1}$ \\ ${ }^{1}$ Universidade Federal de Lavras, Departamento de Engenharia \\ ${ }^{2}$ Universidade Federal de Lavras, Departamento de Física \\ E-mail para contato: tatiane_popiolski@ufla.br
}

\begin{abstract}
RESUMO - Já comercializado no Brasil e amplamente utilizado o Agente Redutor Líquido Automotivo, mais conhecido como Arla 32, é uma solução líquida de alta pureza contendo $32,5 \%$ de ureia e $67,5 \%$ de água deionizada. No Brasil, é utilizado desde 2012 como um redutor de emissão de óxidos de nitrogênio nos sistemas de controle de redução catalítica seletiva (SCR) em veículos pesados movidos a diesel. Arla 32 é uma solução estável, incolor, nãotóxica, não-poluente, não-perigosa e não é inflamável. Não representa nenhum risco grave para os seres humanos, animais, equipamentos ou meio ambiente quando manuseados adequadamente. Com a colaboração universidade-empresa este trabalho teve como objetivo testar uma nova formulação do Agente Redutor Líquido Automotivo com menor teor de ureia. Os resultados obtidos por espectroscopia Raman confirmam a redução do teor de ureia. Novos testes serão realizados para avaliar o desempenho como agente redutor de NOx e compará-lo aos resultados das formulações presentes atualmente no mercado.
\end{abstract}

\section{INTRODUÇÃO}

Diminuir os impactos da emissão de gases tóxicos para a atmosfera é uma das grandes metas da ciência atual. Visando atendê-la o Agente Redutor Líquido Automotivo mais conhecido como Arla 32, uma solução líquida de alta pureza contendo 32,5\% de ureia e $67,5 \%$ água deionizada, estável, incolor, não-tóxica, não-poluente, não-perigosa e não inflamável, é usado desde 2012 como um redutor de emissão de óxidos de nitrogênio nos sistemas de controle de redução catalítica seletiva (SCR) em veículos pesados movidos a diesel no Brasil. Na Europa e EUA, esta tecnologia começou a ser usada a partir de 2006 e é conhecido como AdBlue, DEF (Diesel Exhaust Fluid) ou AUS32 (aqueos urea solution). Atualmente encontram-se muitas ofertas do produto no mercado, sendo vendido pela SHELL, com o SHELL Evolux ARLA 32®; Petrobrás, como Flua Petrobrás®; YARA, comercializado como Air1®, dentre outros.

O sistema SCR equipa motores que em sua combustão priorizam a otimização do consumo de combustível e a redução da formação de material particulado (MP). Esta estratégia ocasiona uma emissão com teores de óxidos de nitrogênio (NOx) mais elevados, acima dos padrões permitidos por lei. Para reduzí-los, o Arla é injetado no sistema de exaustão antes do SCR e transformado em gás de amônia que reage com o NOx na presença 


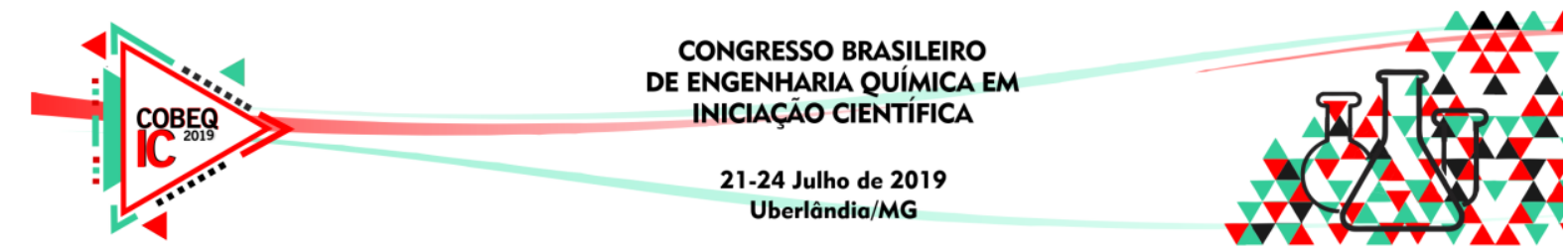

do catalisador de redução seletiva (SCR), produzindo nitrogênio e vapor d'agua, que só então são lançados na atmosfera.

Quando é injetado na corrente de gases de escape quente, a água do Arla evapora e a ureia se decompõe termicamente para formar amônia e ácido isociânico:

$\left(\mathrm{NH}_{2}\right)_{2} \mathrm{CO} \rightarrow \mathrm{NH}_{3}+\mathrm{HNCO}$

O ácido isocianico é hidrolisado em dióxido de carbono e amônia:

$\mathrm{HNCO}+\mathrm{H}_{2} \mathrm{O} \rightarrow \mathrm{CO}_{2}+\mathrm{NH}_{3}$

$\left(\mathrm{NH}_{2}\right)_{2} \mathrm{CO}+\mathrm{H}_{2} \mathrm{O} \rightarrow 2 \mathrm{NH}_{3}+\mathrm{CO}_{2}$

A amônia, na presença de oxigênio e um catalisador, reduzirá os óxidos de nitrogênio:

$4 \mathrm{NO}+4 \mathrm{NH}_{3}+\mathrm{O}_{2} \rightarrow 4 \mathrm{~N}_{2}+6 \mathrm{H}_{2} \mathrm{O}$

$6 \mathrm{NO}_{2}+8 \mathrm{NH}_{3} \rightarrow 7 \mathrm{~N}_{2}+12 \mathrm{H}_{2} \mathrm{O}$

A redução global de NOx por ureia é:

$2\left(\mathrm{NH}_{2}\right)_{2} \mathrm{CO}+4 \mathrm{NO}+\mathrm{O}_{2} \rightarrow 4 \mathrm{~N}_{2}+4 \mathrm{H}_{2} \mathrm{O}+2 \mathrm{CO}_{2}$

$4\left(\mathrm{NH}_{2}\right)_{2} \mathrm{CO}+6 \mathrm{NO}_{2} \rightarrow 5 \mathrm{~N}_{2}+8 \mathrm{H}_{2} \mathrm{O}+4 \mathrm{CO}_{2}$

O objetivo deste trabalho é testar uma nova formulação do Agente Redutor Liquido Automotivo, com menor teor de ureia, o que poderia diminuir o preço da produção do produto, visto que o alto grau de pureza necessário da ureia resulta em um aumento do preço do processo. Inicialmente, deseja-se analisar a composição desta substancia e após isto, seu desempenho como agente redutor de NOx.

\subsection{Produção do Arla}

No Brasil, a produção de Agente Redutor Liquido Automotivo se dá por correntes intermediárias do processo de produção de ureia ou pela dissolução de ureia sólida contendo baixos teores de biureto, $\mathrm{C}_{2} \mathrm{H}_{5} \mathrm{~N}_{3} \mathrm{O}_{2}$, que é resultado da condensação de duas moléculas de ureia (Melo et al, 2015). O biureto é um limitante para a produção do Arla 32, com um teor máximo aceito de 0,3\% (ABNT NBR ISO 22241-1). Acima do limite o biureto forma depósitos no injetor de uréia, conforme reação abaixo: (Leal, 2009).

$2 \mathrm{H}_{2} \mathrm{NCONH}_{2} \leftrightarrow \mathrm{H}_{2} \mathrm{NCONHCONH}_{2}+\mathrm{NH}_{3}$

O processo de produção do Arla divide-se em cinco etapas: síntese, decomposição e absorção, concentração, acabamento e tratamento de efluentes.

A ureia fertilizante e a ureia industrial não são recomendadas para produção do Arla 32, devido aos seus elevados níveis de biureto, $1 \%$ e $0,95 \%$, respectivamente. Além da elevada concentração de formaldeído na uréia fertilizante. 


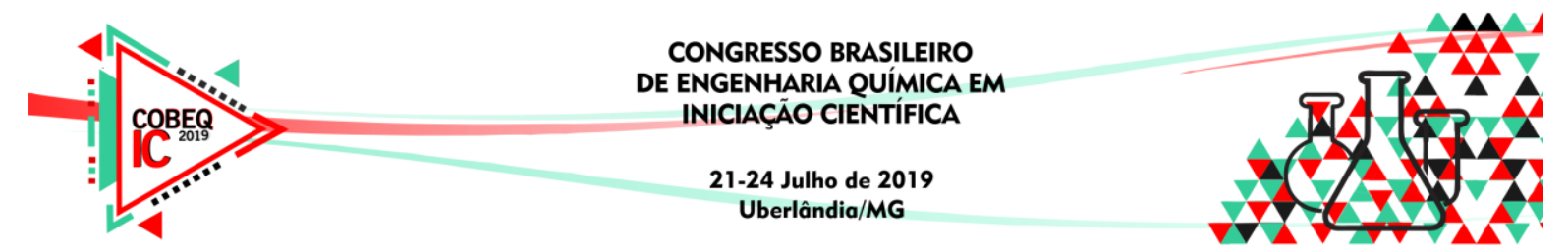

Tendo como referencial o Arla 32 comercial especificado pela BR Distribuidora, Flua ${ }^{\circledR}$, Melo et al (2015) testou quatro diferentes formulações do Arla 32, simulando adulterações:

A. Adição de $10 \%$ de água comum ao Arla 32 de referência;

B. Ureia sólida fertilizante em $67,5 \%$ de água desmineralizada;

C. Ureia sólida fertilizante em $67,5 \%$ de água potável comum;

D. Ureia sólida industrial em $67,5 \%$ de água desmineralizada.

Todas as amostras foram analisadas nos laboratórios da Petrobras para caracterização e comparação com a especificação da NBR ISO 22241-1 (2011).

As análises demonstraram que o Arla Flua está totalmente enquadrado nas especificações da legislação, por outro lado a amostra A apresentou o teor de biureto mais elevado de todas as amostras $(0,4 \%)$ e não atendeu as especificações previstas na legislação. As amostras A e $\mathrm{C}$ apresentaram valores muito elevados de sódio e cálcio devido a água comum, além de que a amostra $\mathrm{C}$ apresentou altas taxas de magnésio e potássio. As formulações feitas a partir de ureia fertilizante não atenderam ao limite de teor de aldeídos Melo et al (2015).

Testes de emissões de NOx também foram realizados onde verificam um aumento de emissão de até $13 \%$ para as formulações que usaram ureia fertilizante e ureia industrial e com relação à formulação que usou um adicional de $10 \%$ de água no Arla 32, o aumento foi de até $32 \%$ Melo et al (2015).

\subsection{Emissão de poluentes}

Segundo uma pesquisa realizada na Universidade de Berkley quase 8 (oito) milhões de pessoas morrem por ano devido a doenças respiratórias. Em regiões com alta concentração de $\mathrm{O}_{3}$ na troposfera, formado por óxidos nitrosos, $\mathrm{NOx}$, e hidrocarbonetos liberados na queima de combutíveis fosseis a probabilidade de mortes por problemas respiratórios aumentam em $30 \%$, e a cada dez pontos percentuais de aumento na concentração de ozônio este índice aumenta em 4\%. (Jerrett et al., 2009).

Desde 2012, visando atender a principal meta da sétima etapa do Proconve (Programa de Controle de Poluição do Ar por Veículos Automotores), baseada no padrão Euro V, que é diminuir as emissões de Óxido de Nitrogênio (NOx) em até 60\% e 80\% de material particulado (MP), duas tecnologias de controle de emissões de motores passaram a ser produzidas e exigidas, o sistema SCR (Selective Catalytic Reduction ou catalisador de redução seletiva) requerendo a utilização do Agente Redutor Líquido de óxidos de nitrogênio Automotivo (Arla32) e o sistema EGR (Exhaust Gas Recirculation ou recirculação de gases de escapamento) combinado com filtro de material particulado ou catalisador de oxidação (Resolução CONAMA, 2008).

\subsection{Funcionamento do Sistema SCR}

O sistema SCR só funciona quando o carro está em movimento. O Arla32 é armazenado em um tanque específico e succionado por uma bomba que o pressuriza a 5 bar e 


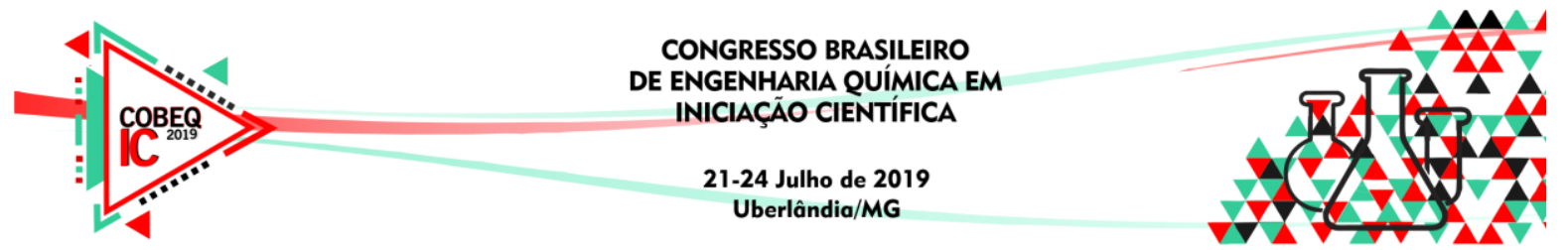

o injeta no sistema de escape por onde passam os gases de combustão provenientes do motor. Quando submetida a altas temperaturas a ureia contida no Agente Redutor se transforma em amônia e se mistura aos gases. Esta mistura é transportada até o catalisador, onde a ureia reage com os óxidos de nitrogenio (NOx), transformado-os em nitrogênio e vapor de água. $\mathrm{O}$ consumo do Agente Redutor é de 4 a $5 \%$ do consumo do diesel.

\section{METODOLOGIA}

Foi analisado a composição do novo Agente Redutor Líquido Automotivo por espectroscopia Raman. Utilizando-se como referencia o Arla 32 comercial especificado pela EUROARLA LTDA, EuroArla ${ }^{\circledR}$. As análises foram conduzidas em um espectofotometro LabRAM HR Evolution, na Universidade Federal de Lavras, do Departamento de Física. Utilizaram-se laser com comprimento de onda de $532 \mathrm{~nm}, 1 \%$ de potência, tempo de 10 segundos e ampliação de 50 vezes.

O tratamento dos dados e as intensidades das bandas foram calculadas através do software OriginPro 8.5, bem como os valores de número de onda correspondentes.

\section{RESULTADOS E DISCUSSÃO}

Ao analisar os dados obtidos por espectroscopia Raman (Figura 1) da amostra tomada como referência, EuroArla ${ }^{\circledR}$, chamada no gráfico de "comercial", pode-se notar dois principais picos o primeiro, "A", em aproximandamente $1000 \mathrm{~cm}^{-1}$ e o segundo, "B", em aproximadamente $3385 \mathrm{~cm}^{-1}$. Os dois principais picos são referentes a ureia e água deionizada, respectivamente.

Figura 1: Espectros Raman da amostra estudada e da referencial

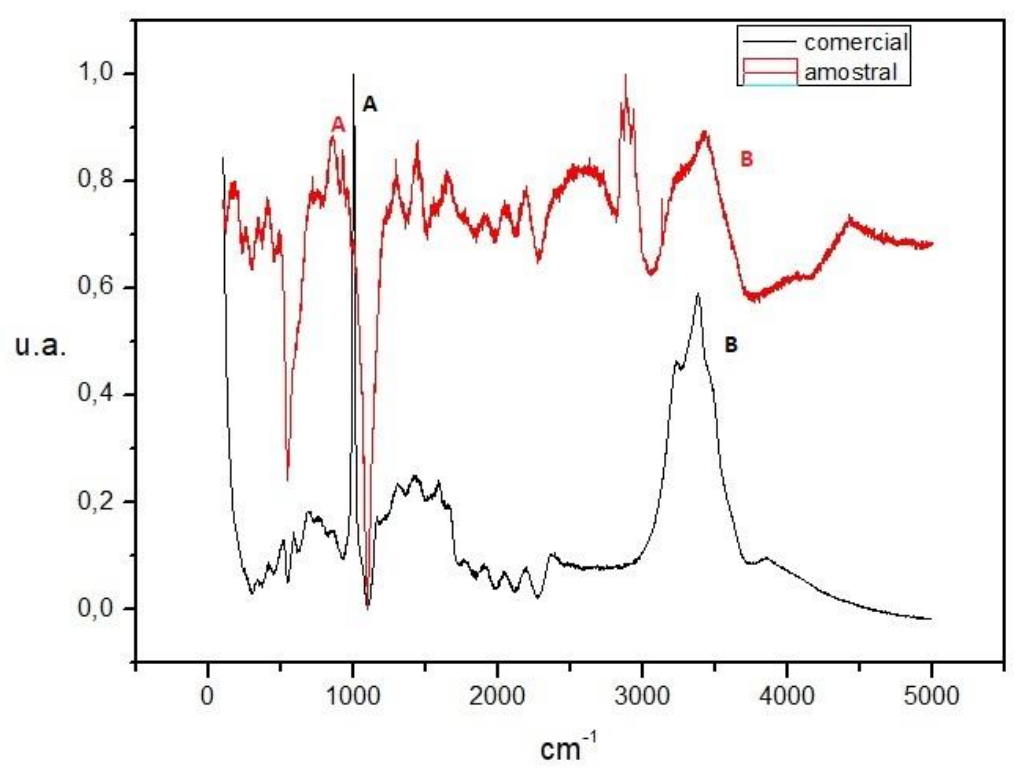




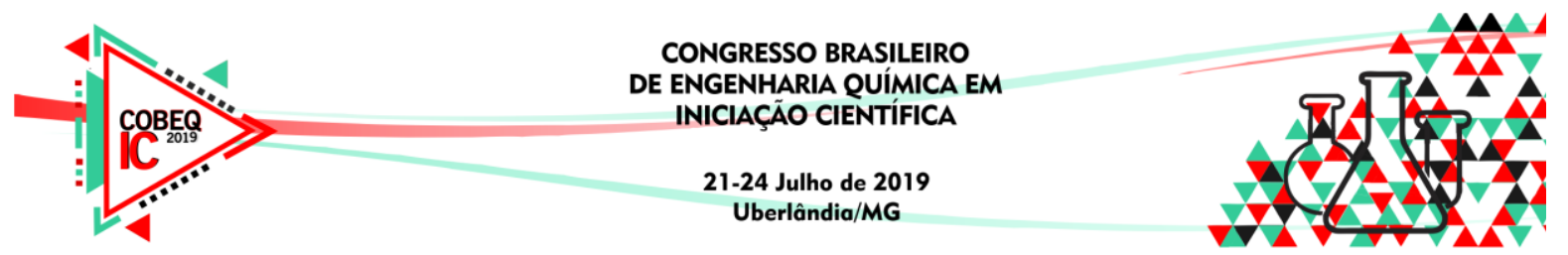

O pico B refere-se à água, segundo Angel et al (2012) e o pico A, à ureia, corroborando com os dados de Saini et al (2014). Os resultados de espectroscopia Raman confirmam a composição da substância fornecida pelo fabricante, EUROARLA LTDA, de 32,5\% de ureia e $67,5 \%$ de água deionizada.

Ao comparar os dados obtidos da amostra referencial e da estudada (figura 1), observase que o pico de água, "B", na amostra estudada possui menor intensidade que na amostra de refenrencia, o que demonstra uma menor quantidade desta substância na formulação da amostra, fator que pode diferi-la das formulações presentes no mercado atualmente.

Em relação a ureia, a análise dos resultados mostram uma menor taxa de ureia no novo agente redutor, o que poderia significar uma redução de custos no processo, porém para confirmar tal suposição serão necessários novos testes que possam indicar os possíveis grupos funcionais presentes nesta amostra, já que ao comparar os dados, percebe-se também a existencia de outros picos distintos em cada uma, o que poderia significar o uso de novas substancias na formulação da amostra estudada.

Uma análise de espectroscopia de infravermelho por transformada de Fourier (FTIR) poderá indicar se na amostra estudada, há grupos funcionais amina, o que seria um indicio da presença de ureia, que juntamente com os dados já obtidos poderá indicar a possível taxa de ureia. Além disso, tal análise também servirá para encontrar outros grupos funcionais presentes, o que será que grande valia, pois se confirmada a existência ou uma menor taxa de ureia, será necessário estudar a nova substância que atua como agente redutor e seu desempenho. Aliado a isto, testes de emissão de NOx serão realizados para o estudo do desempenho desta nova formulação.

\section{CONCLUSÕES}

Os resultados de espectroscopia Raman comprovaram que o novo Agente Redutor é realmente distinto das formulações hoje encontradas no mercado, com uma menor quantidade de água e ureia, a redução desta ultima pode representar uma redução significativa dos custos na produção do Agente Redutor, visto que o alto grau de pureza exigido da ureia para que possa atuar como Agente Redutor sem causar danos ao veículo e ter seu desempenho máximo, o que acarreta em uma elevação dos custos para sua sintese. Os picos encontrados que diferem da amostra referencial, podem indicar novas substâncias utilizadas, as quais, se comprovada sua existência, poderão ser alternativas para a redução da ureia.

\section{REFERÊNCIAS}

ANGEL, S.; GOMER, N.; SHARMA, S.; MCKAY, C.; (2012). Remote Raman Spectroscopy for Planetary Exploration: A Review. Applied spectroscopy. 66. 137-50. 10.1366/1106535.

ABNT NBR ISO 22241-1: 2011 - Motores Diesel - Agente Redutor Líquido Automotivo ARLA 32. Parte 1 - Requisitos de qualidade 2011, ABNT.

JERRETT, M. et al. Long-Term Ozone Exposure And Mortality. Journal of Medicine, New England, v. 360, n. 11, p. 1085-1095, 12 Mar. 2009. 


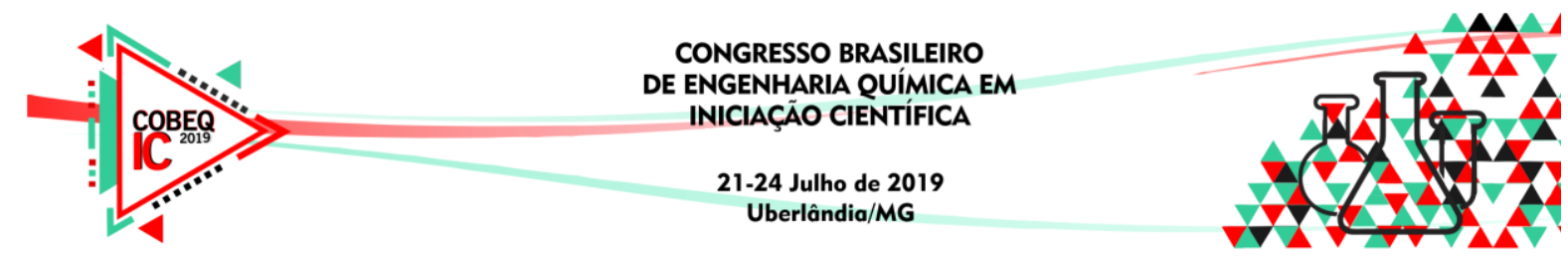

LEAL, G., Seminário ARLA 32 e Seu Papel no Controle da Poluição do Ar, AEA Associação Brasileira de Engenharia Automotiva, SP, 11/08/2009.

MELO, T. C. C.; GIONGO, A.; BRITO, M. F. M.; FREITAS, F. A.; PIRES, L. C.; FURLAN, C. A.; JUNIOR, J. A. D. S.; "Efeito da qualidade da solução de ARLA 32 na eficiência do SCR, nas emissões de NOx e no sistema de injeção de ARLA", p. 399-417 Anais do XXIII Simpósio Internacional de Engenharia Automotica - SIMEA 2014 São Paulo: Blucher, 2015. . São Paulo: Blucher, 2015..

RESOLUÇÃO CONAMA no 403, de 11 de novembro de 2008. MINISTÉRIO DO MEIO AMBIENTE - CONSELHO NACIONAL DO MEIO AMBIENTE - CONAMA. Publicada no DOU n 220, de 12 de novembro de 2008, Seção 1, página 92.

SAINI, A.; MEDWAL, R.; BEDI, S.; MEHTA, B.; GUPTA, R.; MAURER, T.; PLAIN, J.; SUBRAMANIAN, A. (2014). Axonic Au Tips Induced Enhancement in Raman Spectra and Biomolecular Sensing. Plasmonics. 10. 10.1007/s11468-014-9847-4. 